\title{
Current management of pediatric appendicitis: A Central European survey
}

\author{
Vojtech Dotlacil ${ }^{1, A-D, F}$, Barbora Frybova ${ }^{1, B, E}$, Natalie Polívka ${ }^{1, B}$, Daniel Kardos ${ }^{2, B, D, E}$, Peter Vajda ${ }^{2, D, E}$, \\ Krystian Toczewski ${ }^{3, B, C}$, Rebeka Pechanová ${ }^{4, B, C}$, Jozef Babala ${ }^{4, D, E}$, Michal Rygl ${ }^{1, C}{ }^{1, F}$, Dariusz Patkowski ${ }^{3, D, E}$ \\ ${ }^{1}$ Department of Pediatric Surgery, Second Faculty of Medicine, Charles University and Motol University Hospital, Prague, Czech Republic \\ 2 Department of Pediatrics, Surgical Division, University of Pecs, Hungary \\ ${ }^{3}$ Department of Pediatric Surgery and Urology, Wroclaw Medical University, Poland \\ ${ }^{4}$ Department of Pediatric Surgery, Comenius University in Bratislava, National Institute of Children's Diseases, Slovakia \\ A - research concept and design; B - collection and/or assembly of data; C - data analysis and interpretation; \\ $D$ - writing the article; $E$ - critical revision of the article; $F$ - final approval of the article
}

\section{Address for correspondence \\ Vojtech Dotlacil}

E-mail: vojtech.dotlacil@fnmotol.cz

\section{Funding sources}

None declared

\section{Conflict of interest}

None declared

\section{Acknowledgements}

The authors are grateful to all departments that participated in the study. Without their help, this work would not have been possible.

Received on December 19, 2019

Reviewed on January 27,2020

Accepted on May 8, 2020

Published online on June 30, 2020

\section{Cite as}

Dotlacil V, Frybova B, Polívka N, et al. Current management of pediatric appendicitis: A Central European survey. Adv Clin Exp Med. 2020;29(6):745-750. doi:10.17219/acem/122176

DOI

$10.17219 /$ acem/122176

\section{Copyright}

Copyright by Author(s)

This is an article distributed under the terms of the

Creative Commons Attribution 3.0 Unported (CC BY 3.0)

(https://creativecommons.org/licenses/by/3.0/)

\section{Abstract}

Background. Appendicitis is one of the most common diagnoses in pediatric populations. Although new recommendations for the treatment of pediatric appendicitis were published, management varies among different institutions.

Objectives. To determine current practices in $4(n=4)$ representative pediatric surgical departments in Central Europe.

Material and methods. One department from each of the 4 countries was surveyed using an online questionnaire. Questions focused on preoperative, operative and postoperative practices in 2018, particularly those related to antibiotic (ATB) therapy and laparoscopy.

Results. A total of 519 appendectomies were performed, among which 413 (79.6\%) were laparoscopic appendectomies (LAs), with a conversion rate of $5.1 \%$. Appendectomy, as an elective procedure, was performed in 43 (8.3\%) patients. One-quarter (129 patients) had complex appendicitis and $72.3 \%$ of these were operated laparoscopically. In 3 departments, ATB prophylaxis was administered, based on the decisions of the operating surgeon. One department used standard ATB prophylaxis (metronidazole). Whenever phlegmonous appendicitis was detected, ATB were administered therapeutically in 2 departments. Two other departments administered ATB based on surgeon decision. The choice of ATB was not standardized. If complex appendicitis was detected, all sites administered ATB therapeutically. The type of ATB treatment was standardized in complex cases in 2 departments. Thirty-four complications (6.6\%) at surgical sites were recorded - 4.1\% (16/390) after uncomplicated and 14\% (18/129) after complex appendicitis. Thirty-two occurred after acute surgeries and 26 of these followed laparoscopic procedures. Postoperatively, intraabdominal abscesses occurred in 3.5\% of laparoscopic and in $2.9 \%$ of open appendectomy (OA) cases.

Conclusions. This questionnaire study showed that treatment outcomes for appendicitis in children in Central Europe are comparable with data reported in the literature. Laparoscopic appendectomy is the predominant surgical method, but there is a little consensus for ATB treatment in the management of appendicitis at our 4 pediatric surgical departments.

Key words: pediatric surgery, antibiotics, laparoscopy, appendectomy, postoperative complications 


\section{Introduction}

Appendicitis is one of the most common acute surgical diagnoses in children, with appendectomy being the most commonly performed emergency operation. ${ }^{1,2}$ Inflammation of the vermiform appendix ranges from simple irritation to perforation with diffuse peritonitis. Therapy includes a combination of surgical and antibiotic (ATB) treatment. The traditional approach to appendectomy is the open surgical approach - open appendectomy (OA), first described by McBurney in $1894 .{ }^{3}$ Laparoscopic appendectomy (LA) was initially controversial (the first LA was performed in 1983 by Professor Kurt Semm), but recently LA has emerged as standard care in pediatric patients with acute appendicitis. Antibiotics are crucial for the treatment of complex appendicitis, and prophylactically before appendectomies. ${ }^{4}$ Their correct indication is important in reducing unjustified use, optimizing hospitalization stays and maximizing savings while maintaining safe levels of healthcare. ${ }^{5}$ According to evidence-based medicine (EBM), new recommendations have been published for the treatment of pediatric appendicitis in surgical terms and ATB therapy. ${ }^{6}$ This pilot study aimed to determine the current practices in 4 representative pediatric surgical departments in Central Europe.

\section{Material and methods}

A multicenter retrospective questionnaire was addressed to pediatric departments in the Czech Republic, Hungary, Poland, and Slovakia, using an online survey (SurveyMonkey Inc., San Mateo, USA). The questionnaire (Fig. 1) consisted of 10 questions focusing on the type and number of appendicitis operations, surgical approaches, ATB prophylaxes and therapies, and the number of postoperative complications at surgical site infections (SSIs) in 2018. Data was collected anonymously.

\section{Results}

All 4 departments completed the questionnaire. In 2018, 519 appendectomies were performed in participating departments. All departments performed both OA and LA (Fig. 2). Four hundred and thirteen patients (79.6\%) had LA, with conversion in 21 (5.1\%) patients. Appendectomy as an elective procedure (during surgery for recurrent abdominal pain) was performed in 43 (8.3\%) patients. Three-quarters of patients (390) had uncomplicated appendicitis (chronic, catarrhal or inflamed but grossly intact, nongangrenous, nonsuppurative appendix with no associated abscess or peritonitis). The remaining patients (129) had complex appendicitis (gangrene, perforation or peri-appendicular abscess/mass) (Fig. 3).
Thirty-five (OA; 27.1\%) patients were operated by open surgery and 94 (LA; 72.9\%) were operated laparoscopically. One department used standard preoperative ATB treatment (metronidazole), immediately started when the decision to perform an appendectomy was made. At the remaining departments, ATBs were administered according to decisions made by the consultant surgeon. Antibiotics were therapeutically administered in 2 departments, whenever phlegmonous appendicitis was detected. Two other departments administered ATB based on decisions made by the operating surgeon. The choice of ATB was not standardized. If complex appendicitis was determined, all departments administered ATB therapeutically, 2 according to their protocols. Overall, the SSI rate was $6.6 \%(34 / 519)$. Almost all infections occurred after acute surgeries $(94 \%, 32 / 34)$. After surgery for uncomplicated appendicitis, the complication rate was $4.1 \%(16 / 390)$, and $14 \%$ after complex appendicitis (18/129). In terms of acute complications, 7\% (26/373) occurred after LA and 5.8\% (6/103) after OA. In a subgroup of organ-specific complications, abscesses after acute LA were observed in 3.5\% (13/373) of patients and 2.9\% (3/103) of patients after acute OA.

\section{Discussion}

Over the last 2 decades, studies on pediatric appendicitis have been increasing with a focus on the laparoscopic approach, as well as the correct ATB policy for the most effective treatment and reduction of postoperative morbidity. ${ }^{7}$ In our questionnaire study, we focused on the current state of treatment of acute appendicitis in 4 representative pediatric surgical departments in Central Europe.

\section{Laparoscopy}

The data showed that equipment for LA was available in all departments and that laparoscopy had become the method of choice for almost $80 \%$ (413) of patients at the participating departments. The conversion rate was $5.1 \%$. Onequarter of all patients had complex appendicitis (gangrene, perforation or peri-appendicular abscess/mass) and even in these cases, $72.3 \%$ were operated on laparoscopically.

Our results are comparable with the 2017 EUPSA questionnaire, according to which most pediatric surgeons selected a laparoscopic approach (89\% of simplex appendicitis cases and $81 \%$ of perforated ones). ${ }^{8}$ The results of this comparison are even more interesting in terms of the money spent on healthcare, as most studies on the use of laparoscopy in appendectomy come from Western Europe or the USA, where more money goes to healthcare. In 2017, according to Eurostat, health spending per capita was about $€ 2,773$ in the European Union. In this study, in participating countries, this expenditure was almost $€ 1,000$ 
1) Number of all classic appendectomies performed in 2018

1. For acute appendicitis

2. For planned appendectomy (appendectomy performed during surgical exploration of abdominal cavity due to recurrent abdominal pain)

2) Number of all laparoscopic appendectomies performed in 2018

1. For acute appendicitis

2. For planned appendectomy (appendectomy performed during surgical exploration of abdominal cavity due to recurrent abdominal pain)

3) Number of complicated appendicitis in 2018 (gangrenous, perforated) - according to the perioperative finding - write the absolute number performed for the $1^{\text {st }}$ laparoscopic, for the $2^{\text {nd }}$ classical

4) Number of conversions from laparoscopic to classic appendectomy in 2018

5) Do you use antibiotic (ATB) prophylaxis before appendectomy (antibiotic prophylaxis = preoperative administration of one ATB dose 0-60 min before surgery)?

1. Yes, in all cases

2. Yes, but only in laparoscopic acute appendectomy

3. Yes, but only in classic acute appendectomy

4. Yes, but only in laparoscopic planned appendectomy

5. Yes, but only in the classic planned appendectomy

6. According to the decision of the surgeon

7. No, we do not use ATB prophylaxis

6) Type of ATB prophylaxis (if you use multiple combinations, choose them)

1. Amoxicillinum/acidum clavulanicum

2. Amoxicillinum/acidum clavulanicum + gentamicin

3. Amoxicillinum/acidum clavulanicum + metronidazole

4. Amoxicillinum/acidum clavulanicum + gentamicin + metronidazole

5. Piperacillinum/tazobactamum

6. Other type or combination of ATB - please list

7) Do you use ATB postoperatively in the case of phlegmonous appendicitis?

1. Yes

2. No

3. According to the surgeon

4. If yes, what (please list and specify dosing schedule)

8) Do you use ATB postoperatively in case of gangrenous appendicitis?

1. Yes

2. No

3. According to the surgeon

4. If yes, what (please list and specify dosing schedule)

9) Do you use ATB postoperatively in case of perforated (peritonitis, abscess) appendicitis?

1. Yes

2. No

3. According to the surgeon

4. If yes, what (please list and specify dosing schedule protocol)

Duration Way of administration

10) Number of postoperative complications up to the $30^{\text {th }}$ postoperative day - SSI (surface, deep, organ specific - according to CDC - Centers for Disease Control and Prevention)

1. LAA (laparoscopic acute appendectomies) Surface/Deep/Organ-specific

2. LPA (laparoscopic planned appendectomies) Surface/Deep/Organ-specific

3. CAA (classic acute appendectomies)

Surface/Deep/Organ-specific

4. CPA (classic planned appendectomies)

Surface/Deep/Organ-specific 


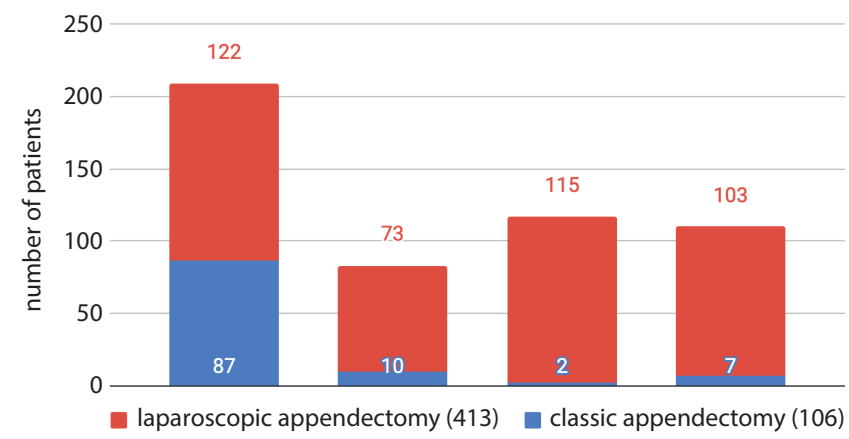

Fig. 2. The number of laparoscopic and classic appendectomies performed at participating departments $(n=4)$

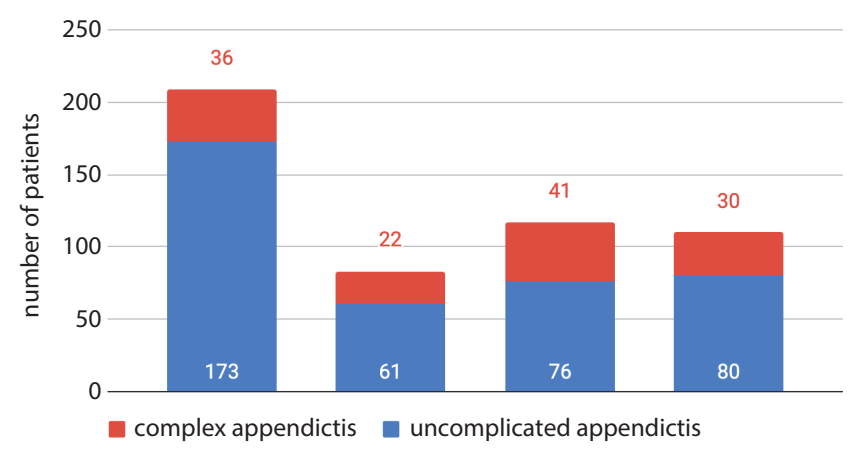

Fig. 3. The number of complex and uncomplicated appendicitis performed at participating departments $(n=4)$

lower (the Czech Republic: $€ 1873$, Hungary: $€ 1473$, Poland: $€ 1409$, and Slovakia: €1625). ${ }^{9}$

Laparoscopic appendectomy in children has been used for over 25 years - its first use was described in 1992 by Ure et al. ${ }^{10}$ According to recent studies and meta-analyses, LA in children shortens the length of hospitalization, and reduces the risk of wound infections and postoperative pain. ${ }^{11-13}$ Furthermore, favorable cosmetic results after LA have also been demonstrated in children. ${ }^{14}$

\section{Antibiotic prophylaxis and treatment}

Antibiotics are effective both prophylactically before an appendectomy to reduce postoperative complications, and in the treatment of complex appendicitis, either following surgery, or as a first choice in the treatment of periappendicular mass or abscess.

Our results showed that ATB prophylaxis was a standard part of treatment in only 1 department, where metronidazole was started preoperatively. The other departments administered ATB according to decisions made by the surgeon. Antibiotic was administered in 2 of the departments whenever a perioperative finding of phlegmonous appendicitis was determined, whereas, in the other 2 , the decision was left to the surgeon. When complex appendicitis was found, ATBs were always administered; in 2 departments according to standard protocols, but the ATB type varied in each department. Some departments still chose a triple ATB combination in case of perforation (Fig. 4).

The use of any drugs, including ATBs, can in extreme cases lead to prescription errors. Excessive indications or inappropriate duration of administration can prolong treatment, hospitalization, cause of complications, increase ATB resistance, and in general, increase the overall cost of treatment. ${ }^{15}$ In 2010, based on a systems review, the American Pediatric Surgery Association's recommendations on prophylaxis and treatment of acute appendicitis were published. ${ }^{16}$ According to this publication, ATB prophylaxis is always recommended before appendectomy, and when uncomplicated appendicitis is found, ATB therapy is not continued (the number of postoperative complications has not decreased with continued ATB administration). In cases of complex appendicitis, the use of broad-spectrum ATBs is always recommended. They should be used either as a monotherapy or in combination with other ATBs (e.g., piperacillin/tazobactam + metronidazole). Previous, standard ATB triple combinations

ABT therapy in complex appendictis

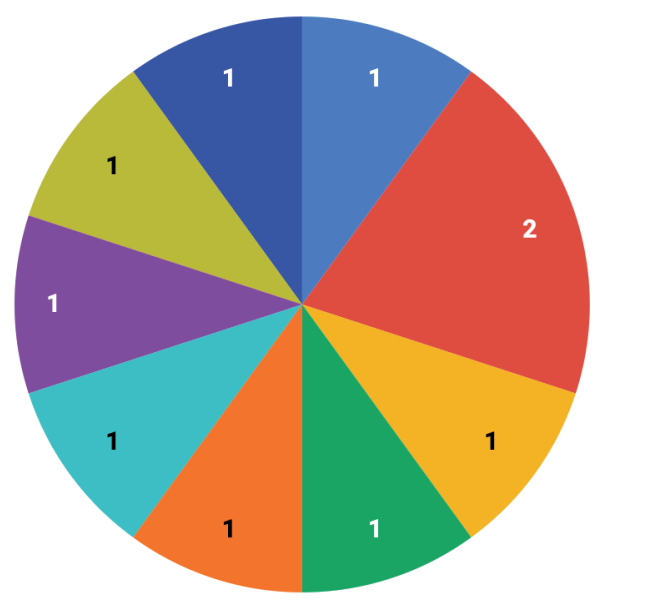

amoxicillinum/acidum clavulanicum + gentamicin

amoxicillinum/acidum clavulanicum + metronidazol

clindamycin

metronidazol

ceftriaxon + metronidazol

amoxicillinum/acidum clavulanicum + cefuroxim + metronidazol

amoxicillinum/acidum clavulanicum + gentamicin + metronidazol

cefuroxim + metronidazol

cefotaximt + gentamicint + metronidazol

Fig. 4. Wide variation in the use of ATB across departments for complex appendicitis 
against Gram-positive, Gram-negative and anaerobic bacteria are no longer recommended. ${ }^{17,18}$

For phlegmonous appendicitis, all departments in certain cases ( 2 always, 2 decided by the surgeon) continued with ATB as therapy and did not follow current EBM recommendations. In complex appendicitis cases, according to current EBM recommendations, 100\% of departments always indicated ATB, but ATB choices and combinations differed greatly. However, inconsistencies in using ATB as a treatment in these departments may reflect a lack of implementation of recent recommendations, and also they may be partly based on their recommendations and on local microbiological findings and recommendations of ATB centers.

Based on our surgical data and literature recommendations, 2 centers developed a uniform ATB prophylaxis and therapy scheme during 2018.

\section{Complications}

Overall, there were 34 (6.6\%) complications; $4.1 \%$ after uncomplicated appendicitis and 14\% after complex appendicitis, and almost all occurred after acute surgeries. Approximately 7\% (26/373) of complications after acute surgeries were noted following laparoscopic procedures, and $5.9 \%(6 / 103)$ were noted following an open procedure. The incidence of intra-abdominal abscesses was 3.5\% (13/373) after acute LAs and 2.9\% (3/103) after acute OAs.

In 2017 a meta-analysis of randomized controlled trials with 3,642 patients (1810 LA and 1832 OA) reported 9.5\% postoperative complications after LA, and $14.8 \%$ after $\mathrm{OA}$, and concluded no significant differences between the 2 groups $(\mathrm{OR}=0.74,95 \% \mathrm{CI}=0.34-1.59, \mathrm{p}=0.44) .{ }^{19}$ In an extensive literature review from 2012, Markar et al. describing 107,624 patients, laparoscopic approaches did not increase the risk of postoperative complications, and in a sub-analysis of patients undergoing LA for complex appendicitis, the risk of postoperative complications was significantly lower. ${ }^{20}$ In a 2012 retrospective study in 1,205 patients after appendectomy, investigating the development of postoperative complications and intra-abdominal abscesses in relation to surgical techniques, comparisons were made for uncomplicated and complex appendicitis, with the conclusion that the type of surgical approach did not affect the development of intra-abdominal abscesses (LA, 3.9\%(19/491) vs OA, 3.9\% (28/714); p = 1.0). ${ }^{21}$ Some surgeons chose the open approach for complex appendicitis because of the greater risk of complications following LA, but this remains unconfirmed in these studies. Some studies have shown the contrary; LA led to a significantly lower number of readmissions ( $1.3 \%$ vs $12.3 \%$; $\mathrm{p}=0.006)$ and reoperations ( $4 \%$ vs $17.2 \%$; $\mathrm{p}=0.006$ ), and significantly lower numbers of postoperative wound complications $(0 \%$ vs $11.5 \%$; $=0.001) .{ }^{22}$ Similar conclusion were reached in the study by Chang et al. who reported that the risk of wound infection was significantly higher in the OA group (12.6\% vs $4 \%$; $\mathrm{p}=0.032) .{ }^{23}$

\section{Conclusions}

This questionnaire study showed that our results of appendicitis treatment in children in Central Europe are comparable with the literature. Laparoscopic appendectomy is the predominant surgical method; however, there appears to be little consensus on ATB treatment for the management of appendicitis at surveyed pediatric surgical departments in Central Europe.

\section{ORCID iDs}

Vojtech Dotlacil @ https://orcid.org/0000-0002-7291-7323 Barbora Frybova (1) https://orcid.org/0000-0001-5744-9747 Natalie Polívka (1) https://orcid.org/0000-0001-7413-5600 Daniel Kardos (10 https://orcid.org/0000-0003-4863-1445 Peter Vajda (1) https://orcid.org/0000-0002-4835-6458 Krystian Toczewski (10 https://orcid.org/0000-0003-4336-2151 Rebeka Pechanová (10 https://orcid.org/0000-0002-8310-9436 Jozef Babala (1) https://orcid.org/0000-0003-1073-9733 Michal Rygl (1) https://orcid.org/0000-0002-4804-039X Dariusz Patkowski (1) https://orcid.org/0000-0002-4583-5517

\section{References}

1. Wan MJ, Murray K, Ungar WJ, et al. Acute appendicitis in young children: Cost-effectiveness of US versus CT in diagnosis. A Markov decision analytic model. Radiology. 2009;250(2):378-386. doi:10.1148/ radiol. 2502080100

2. Buckius MT, McGrath B, Monk J, et al. Changing epidemiology of acute appendicitis in the United States: Study period 1993-2008. J Surg Res. 2012;175(2):185-190. doi:10.1016/j.jss.2011.07.017

3. McBurney $C$. The incision made in the abdominal wall in cases of appendicitis, with a description of new method of operating. Ann Surg. 1894;20:38-43. doi:10.1097/00000658-189407000-00004

4. Shawyer AC, Hatchell AC, Pemberton J, et al. Compliance with published recommendations for postoperative antibiotic management of children with appendicitis: A chart audit. J Pediatr Surg. 2015;50(5); 783-785. doi:10.1016/j.jpedsurg.2015.02.040

5. Fraser JD, Aguayo P, Leys CM, et al. A complete course of intravenous antibiotics vs a combination of intravenous and oral antibiotics for perforated appendicitis in children: A prospective, randomized trial. J Pediatr Surg. 2010;45(6):1198-1202. doi:10.1016/j.jpedsurg. 2010.02.090

6. Lee SL, Islam S, Cassidy LD, et al. Antibiotics and appendicitis in the pediatric population: An American pediatric surgical association outcomes and clinical trials committee systematic review. J Pediatr Surg. 2010;45(11):2181-2185. doi:10.1016/j.jpedsurg.2010.06.038

7. Andersen BR, Kallehave FL, Andersen HK. Antibiotics versus placebo for prevention of postoperative infection after appendectomy. Cochrane Database Syst Rev. doi:10.1002/14651858.CD001439.pub2

8. Zani A, Hall N, Rahman A, et al. European Paediatric Surgeons' Association Survey on the management of pediatric appendicitis. Eur J Pediatr Surg. 2019;29(01):53-61. doi:10.1055/s-0038-1668139

9. OECD/EU (2018), Health at a Glance: Europe 2018: State of Health in the EU Cycle, OECD Publishing, Paris. https://doi.org/10.1787/health_ glance_eur-2018-en

10. Ure BM, Spangenberger W, Hebebrand D, et al. Laparoscopic surgery in children and adolescents with suspected appendicitis: Results of medical technology assessment. Eur J Pediatr Surg. 1992;2(6):336-340.

11. Masoomi H, Mills S, Dolich MO, et al. Comparison of outcomes of laparoscopic versus open appendectomy in children: Data from the Nationwide Inpatient Sample (NIS), 2006-2008. World J Surg. 2012; 36(3):573-578.

12. Jaschinski T, Mosch ChG, Eikermann M, et al. Laparoscopic versus open surgery for suspected appendicitis. Cochrane Database Syst Rev. doi:10.1002/14651858.CD001546.pub4

13. Esposito C, Calvo Al, Castagnetti M, et al. Open versus laparoscopic appendectomy in the pediatric population: A literature review and analysis of complications. J Laparoendosc Adv Surg Tech A. 2012;22(8): 834-839. 
14. Chandler NM, Ghazarian SR, King TM, et al. Cosmetic outcomes following appendectomy in children: A comparison of surgical techniques. J Laparoendosc Adv Surg Tech A. 2014;24(8):584-588.

15. Ameer A, Dhillon S, Peters $M$, et al. Systematic literature review of hospital medication administration errors in children. Integr Pharm Res Pract. doi:10.2147/IPRP.S54998

16. Lee SL, Islam S, Cassidy LD, et al. Antibiotics and appendicitis in the pediatric population: An American Pediatric Surgical Association outcomes and clinical trials committee systematic review. J Pediatr Surg. 2010;45(11):2181-2185. doi:10.1016/j.jpedsurg.2010.06.038

17. Peter ST, Shawn D, Kuojen T, et al. Single daily dosing ceftriaxone and metronidazole vs standard triple antibiotic regimen for perforated appendicitis in children: A prospective randomized trial. $J$ Pediatr Surg. 2008;43(6):981-985. doi:10.1016/j.jpedsurg.2008.02.018

18. Dreznik Y, Feigin E, Samuk I, et al. Dual versus triple antibiotics regimen in children with perforated acute appendicitis. Eur J Pediatr Surg. 2018;28(06):491-494. doi:10.1055/s-0037-1606847
19. Dai L, Shuai J. Laparoscopic versus open appendectomy in adults and children: A meta-analysis of randomized controlled trials. United European Gastroenterol J. 2016;5(4):542-553. doi:10.1177/2050 640616661931

20. Markar SR, Blackburn S, Cobb R, et al. Laparoscopic versus open appendectomy for complicated and uncomplicated appendicitis in children. J Gastrointest Surg. 2012;16(10):1993-2004. doi:10.1007/ s11605-012-1962-y

21. Nataraja RM, Teague WJ, Galea J, et al. Comparison of intraabdominal abscess formation after laparoscopic and open appendicectomies in children. J Pediatr Surg. 2012;47(2):317-321. doi:10.1016/j.jpedsurg. 2011.11.023

22. Vahdad MR, Troebs RB, Nissen M, et al. Laparoscopic appendectomy for perforated appendicitis in children has complication rates comparable with those of open appendectomy. J Pediatr Surg. 2013;48(3): 555-561. doi:10.1016/j.jpedsurg.2012.07.066

23. Chang HK, Han SJ, Choi SH, et al. Feasibility of a laparoscopic approach for generalized peritonitis from perforated appendicitis in children. Yonsei Med J. 2013;54(6):1478-1483. doi:10.3349/ymj.2013.54.6.14 